\title{
Effect of the boundary conditions, temporal, and spatial resolution on the pressure from PIV for an oscillating flow
}

\author{
N. Sakib ${ }^{1 *}$, A. Mychkovsky ${ }^{2}$, J. Wiswall ${ }^{2}$, R. Samaroo ${ }^{2}$, B. L. Smith ${ }^{1}$ \\ ${ }^{1}$ Utah State University, Department of Mechanical and Aerospace Engineering, Logan-UT, USA \\ ${ }^{2}$ Naval Nuclear Laboratory, West Mifflin-PA and Schenectady-NY, USA \\ *nazmus.sakib@usu.edu
}

\begin{abstract}
The pressure field of an impinging synthetic jet has been computed from time-resolved, three-dimensional, three-component (3D-3C) particle image velocimetry (PIV) velocity field data using a Poisson equationbased pressure solver. The pressure solver used in this work can take advantage of the temporal derivative of the pressure to enhance the temporal coherence of the calculated pressure field for time-resolved velocity data. The reconstructed pressure field shows sensitivity to the implementation of the boundary conditions, as well as to the spatial and temporal resolution of the PIV data. The pressure from a 3D Poisson solver that does not consider the temporal derivative of the pressure shows high random error. Invoking the temporal derivative of the pressure eliminates this high-frequency noise, however, the calculated pressure exhibits an unphysical temporal drift. This temporal drift is affected by both the temporal resolution of the PIV data and the spatial resolution of the PIV vector field, which was systematically evaluated by downsampling the instantaneous data and increasing the interrogation window size. It was observed that decreasing the temporal resolution increased the drift, while decreasing the spatial resolution decreased the drift.
\end{abstract}

\section{Introduction}

The pressure field in a flowing fluid is an important dynamic property as this quantity can be used to analyze surface loads and other hydrodynamic conditions. Traditionally, pressure measurements have been limited to surface pressure measurements, where conventional taps or microphone transducers are used to measure the static or fluctuating values, respectively (Van Oudheusden, 2013). Acquiring pressure measurements within the flow field is considerably more difficult than collecting surface pressure measurements by traditional means. Physical pressure probes are intrusive, must be oriented properly with respect to the unknown flow direction, and can only provide local point measurements. The use of physical sensors limits the ability to capture the dynamic aspects of the flow as pressure field information is limited to either coarse, instantaneous measurements provided by a probe array or ensemble-mean statistics provided by a traversing probe (Tsuji et al., 2007).

PIV is an established, non-intrusive flow field measurement technique. With advancements in imaging technology and data processing methods, the accuracy and resolution of PIV has evolved to the level where calculation of derived flow quantities, such as vorticity or divergence, are possible. Volumetric PIV techniques, such as tomographic (tomo-) PIV (Elsinga et al., 2006) and Shake-the-Box (STB) (Schanz et al., 2016), have been developed to enable time-resolved three-dimensional, three-component (3D-3C) measurements to fully characterize the fluid velocity field.

Reconstruction of a pressure field from PIV data was first investigated by Jensen et al. (2001). The method has since been applied to obtain measurements of pressure fluctuations in a turbulent boundary layer (Ghaemi et al., 2012), pressure on an airfoil (Violato et al., 2011), and pressure loads on wind turbine blades (Villegas and Diez, 2014; Lignarolo et al., 2014). PIV-based pressure reconstruction has also been used for biological fluid dynamics problems, including embryonic heart (Bark et al., 2017) and glottal channel studies (Oren et al., 2015). 
Though PIV-based pressure reconstruction is now considered to be a quantitative measurement technique, assessments of the uncertainty and its dependence on various experimental and numerical parameters, namely the spatial and temporal resolution and the boundary conditions, have been limited. It has been shown theoretically that for a 2D pressure Poisson solver, the propagation of Gaussian errors from the velocity field to the pressure field is affected by the shape and area to volume ratio of the flow domain, boundary conditions, and the velocity error level in the field and in the boundary (Pan et al., 2016). The work by Pan et al. (2018) also shows that for Poisson equation based pressure solvers, the numerical truncation error is dominant in coarse spatial resolution and the experimental error is dominant in fine spatial resolution.

Temporal resolution must also be considered for Poisson equation based pressure solvers that use Lagrangian tracking to estimate the material acceleration of the fluid. A recent Poisson equation based pressure solver (Jeon et al., 2017) uses the convection velocity of the flow structures to calculate the temporal derivative of pressure and then compute the pressure fields from time resolved PIV data. The pressure solver uses boundary conditions of pressure at specific boundary points or as an average pressure in a specified volume in the measurement domain. Except for the locations of the specific pressure, Neumann type boundary conditions are used for all other boundary points. The boundary conditions can be provided either at the beginning of the time series or as constant across every time step.

In this work, time-resolved tomo-PIV measurements of an impinging synthetic jet in a water filled tank are used to calculate the pressure field using a Poisson equation based pressure solver. The periodic nature of the synthetic jet velocity field makes it an ideal case for studying PIV-based pressure reconstruction results as one can expect the pressure at any location to repeat each cycle. The effect of different implementations of the boundary conditions is evaluated by directly comparing the pressure calculated from PIV at a particular surface location with that obtained from a pressure sensor at the same location. In addition, the effect of temporal and spatial resolution (relative to the major flow scale) on the calculated pressure field is examined by varying the frequency and the displacement amplitude of the synthetic jet.

\section{Pressure from PIV}

A common method for calculating a pressure field from PIV data is summarized in (Van Oudheusden, 2013). This technique uses measured velocity fields to calculate the pressure gradient within the flow. The pressure field is then calculated from the pressure gradient with the appropriate boundary conditions. Section 2.1 explains the general approach for computing a fluid pressure field from a measured velocity field while section 2.2 describes the pressure solver algorithms used in this study.

\subsection{Fluid pressure from a Measured Velocity Field}

The relationship between velocity and pressure in a flow field is described by the Navier-Stokes momentum equation,

$$
\nabla p=-\rho \frac{D \mathbf{u}}{D t}+\mu \nabla^{2} \mathbf{u}
$$

where $p, \mathbf{u}, t, \rho$, and $\mu$ denote pressure, velocity, time, density, and dynamic viscosity of the fluid, respectively. The material acceleration, $\frac{D \mathbf{u}}{D t}$ can be expressed in an Eulerian frame of reference as

$$
\frac{D \mathbf{u}}{D t}=\frac{\partial \mathbf{u}}{\partial t}+(\mathbf{u} \cdot \nabla) \mathbf{u}
$$

The pressure field can be obtained by spatial integration of the pressure gradient (equation 11) (Liu and Katz, 2004). However there are numerical challenges associated with this approach. This study focuses on the more common Poisson equation based pressure solver. The pressure Poisson equation is derived by applying the divergence operator to equation 1 and substituting equation 2 for the material acceleration to yield

$$
\nabla^{2} p=-\nabla \cdot((\mathbf{u} \cdot \nabla) \mathbf{u}) .
$$

Both the viscous and temporal terms are eliminated with continuity for an incompressible flow $(\nabla \cdot \mathbf{u}=0)$, and the pressure field can be calculated from the velocity field using pressure boundary conditions.

In practice, it can be challenging to determine the pressure boundary conditions to calculate the pressure field using equation 3. Pressure measurements can be used to determine Dirichlet (pressure as a function of 
time at a spatial location) boundary conditions; however, in general, instantaneous measurements synchronized with the instantaneous velocity fields must be obtained at multiple spatial locations, and in many cases it is not feasible to determine the pressure boundary conditions using only pressure measurements. To address the problem of determining pressure boundary conditions, additional information can be determined from the measured velocity fields. Specifically, if the material derivative can be determined from several instantaneous velocity fields, a Neumann boundary condition (pressure gradient as a function of time at a spatial location) can be calculated using the Navier-Stokes equation, and the pressure field can be calculated using a combination of Dirichlet and Neumann boundary conditions.

When using the velocity field data to calculate pressure boundary conditions, both the spatial derivative terms of the velocities within the domain using equation 3 and the pressure gradients at the boundary conditions using equation 1 are calculated. Because the material acceleration is calculated, the pressure solver may first calculate the pressure gradient throughout the entire measurement domain from the NavierStokes equation using the material acceleration term, and then use the following identity to spatially integrate the pressure gradient

$$
\nabla^{2} p=\nabla \cdot(\nabla p)
$$

\subsection{Pressure Solver Algorithms Used in This Study}

The pressure solver in LaVision DaVis 10.2 was used in this work. This software employs iterative pseudoLagrangian tracking to calculate the material acceleration (Liu and Katz, 2006). The position of the same group of tracer particles located at the grid points are tracked over multiple vector fields to compute the velocity and material acceleration using a polynomial trajectory model. The computation cost of material acceleration calculation increases with the number of velocity fields considered. In this study 5 velocity fields were used to estimate the velocity and material acceleration. The calculated material acceleration is then optimized in a least squares manner. There are several other methods to calculate the material acceleration from PIV velocity fields such as the Eulerian approach (Baur and Kongeter, 1999), Taylor's hypothesis approach (De Kat and Ganapathisubramani, 2013; Laskari et al., 2016) and the instantaneous vortex-in-cell (VIC) method (Schneiders et al., 2016). The iterative pseudo-Lagrangian approach and the Eulerian approach are purely numeric and therefore require time-resolved data with at least two subsequent velocity fields as an input to calculate the material acceleration while the Taylor hypothesis approach and the VIC method incorporate physical flow models so that a single, instantaneous velocity field is sufficient.

Once the material acceleration is calculated the pressure gradient is obtained by adding the viscous terms from equation 1 , and is integrated with a pressure Poisson solver to obtain the pressure field. The LaVision solver used in this study uses a modified operator (equation 5) that includes the time derivative of pressure to calculate the pressure fields from time-resolved PIV velocity fields.

$$
\nabla^{\prime 2} p=\frac{\partial^{2} p}{\partial x^{2}}+\frac{\partial^{2} p}{\partial y^{2}}+\frac{\partial^{2} p}{\partial z^{2}}+\left.\xi \frac{\partial^{2} p}{\partial t^{2}}\right|_{C}
$$

where $\nabla^{\prime 2}$ is a modified operator and $\xi$ is the weighting factor between the temporal and spatial derivative of the pressure. This approach eliminates the requirement of at least one Dirichlet boundary condition per each time-step except the very first time-step.

The time derivative of pressure is estimated by making the assumption that the pressure does not change on the convective frame which provides the following expression for the temporal derivative of the pressure (Jeon et al., 2017)

$$
\left.\frac{\partial p}{\partial t}\right|_{C} \approx \frac{1}{\Delta t} \int_{x+\mathbf{u}_{c} \Delta t}^{x} \nabla p d \mathbf{x}
$$

where $\mathbf{u}_{c}$ is the estimated convection velocity of the vortical structures. Additionally, the solver allows the temporal derivative term in equation (5) to be omitted by setting $\xi$ to a small value. In this case, the convection velocity of the flow structure is not calculated and is equivalent to computing the pressure field separately for each of the instantaneous velocity fields. Taking the time derivative of pressure to be zero is similar to computing the pressure field separately for each of the velocity fields. The weighting factor $\xi$ allows studying the effect of the enforcement of strong or weak temporal coherence on the calculated pressure. Considering the modified operator, equation 4 takes the following form

$$
\nabla^{\prime 2} p=f_{\text {exp }}
$$

where the $f_{\text {exp }}$ term is calculated from the experimental velocity field. 


\section{Experiment}

The experimental facility used in this work is described in section 3.1 and the experimental parameter space is outlined in Section 3.2 .

\subsection{Experimental Facility and Measurement}

Figure 1 shows a schematic diagram of the experimental facility, which consists of a synthetic jet impinging on a circular plate contained in a hexagonal water-filled tank. The hexagonal sides of the aquarium act as water prisms to improve the camera viewing angles. An electromagnetic shaker oscillates a piston inside of a cylinder and pushes water through an orifice in the bottom surface of the tank, producing a synthetic jet, which generates vortex rings that travel upwards through the stagnant water in the tank towards the impingement surface.

The impingement plate is fitted with three pressure sensors to provide capability for both Dirichlet boundary conditions and validation measurements for the pressure calculated from PIV as shown in figure 1 . In the initial experimental arrangement, the jet was positioned near the right edge of the measurement volume. However, this setup allows the vortices to cross the boundary of the measurement volume as it propagates upwards which subsequently intensifies the temporal drift that is discussed below in section 4.1. To avoid the high temporal drift of the calculated pressure, the setup was modified to place the jet near the center of the measurement volume.

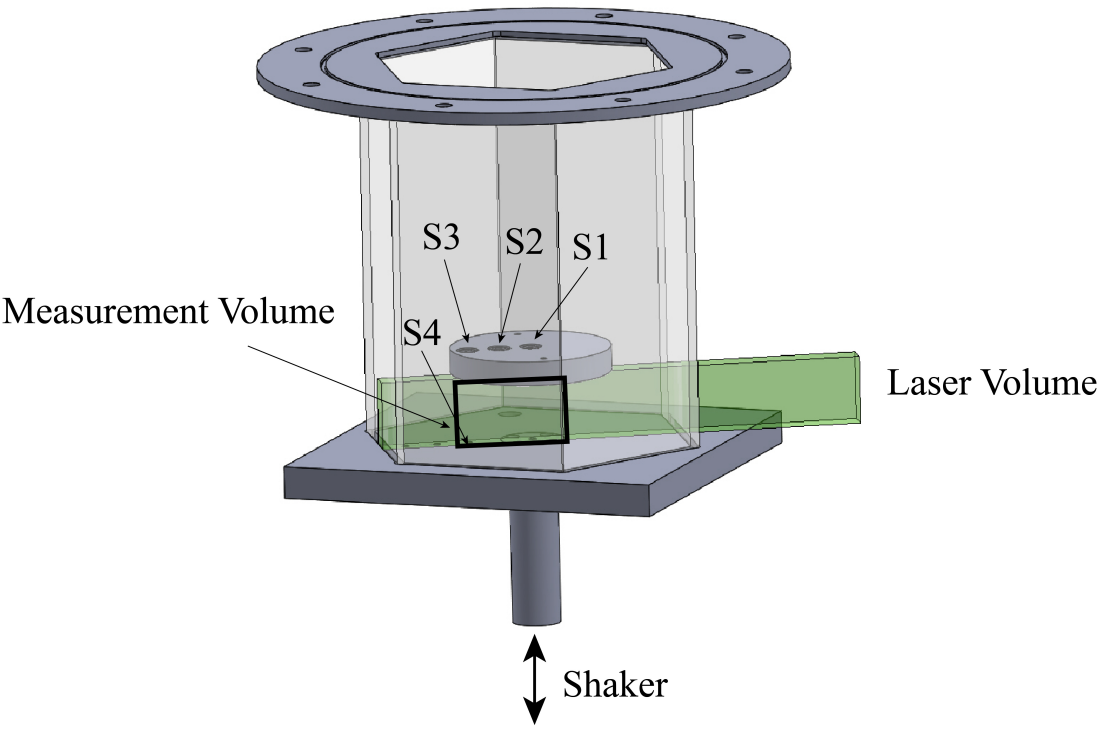

Figure 1: Schematic diagram of the experimental facility. The thickness of the measurement volume is 20 $\mathrm{mm}$. S1, S2 and S3 are the locations of the pressure sensors. The S1 pressure sensor is at the impingement point.

Frame-straddled, time-resolved images of the flow field were captured with four high-speed CMOS (Complementary Metal-Oxide-Semiconductor) cameras. The flow was seeded with neutrally buoyant phosphorescent micro plastic particles of $50 \mu \mathrm{m}$ diameter. A measurement volume of $60 \mathrm{~mm} \times 55 \mathrm{~mm} \times 22 \mathrm{~mm}$ was illuminated by a dual-cavity Nd:YLF (Neodymium-doped Yttrium Lithium Fluoride) laser. The raw images from the four cameras were processed by a multi-pass, 3D cross-correlation algorithm with a final interrogation volume of $32 \times 32 \times 32$ voxels. A $75 \%$ interrogation volume overlap resulted in a vector spacing of $0.41 \mathrm{~mm}$. The temporal resolution of the PIV data is $1 \mathrm{~ms}$. The velocity field obtained with tomographic PIV is well resolved to capture the flow features of the synthetic jet propagation and impingement. 
(a)

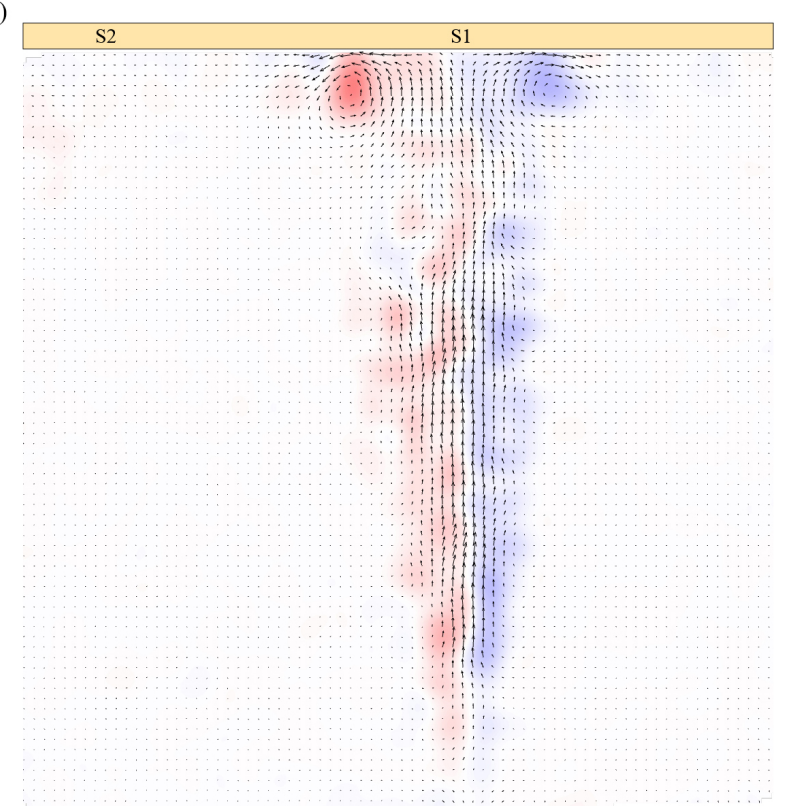

(a) (b)

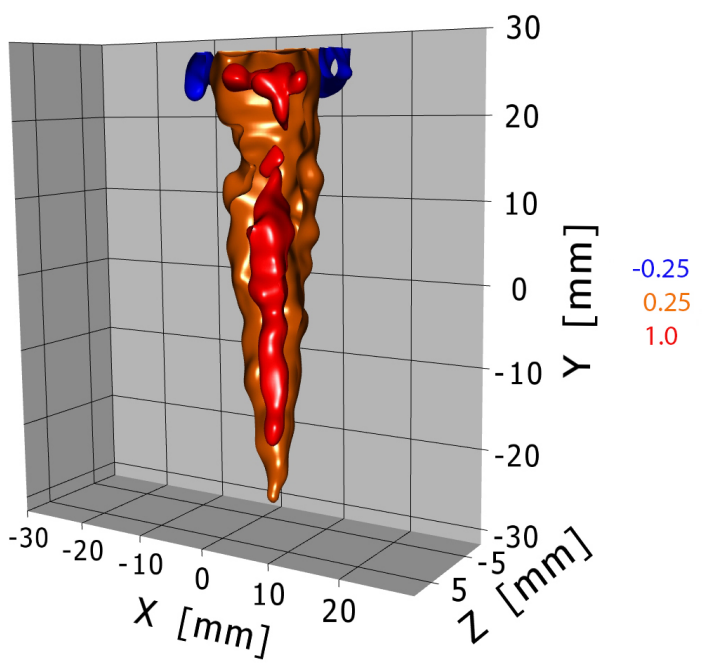

(b)

Figure 2: The velocity field obtained by tomographic PIV. The synthetic jet has a displacement amplitude of $40 \mathrm{~mm}$. (a) Slice of the vector field at the mid $x y$-plane. The background colors represent the out of plane vorticity with red and blue denoting the counter clockwise and the clockwise motion respectively. (b) Iso surfaces of the streamwise velocity in 3D.

\subsection{Experimental Parameter Space}

The test facility was operated to produce three synthetic jets with similar velocities and therefore similar pressure, but unique frequencies and displacement amplitudes, $L_{0}$ (the distance traveled by a fluid particle during the forward stroke of the cycle). This enabled the effective temporal resolution of the PIV measurements relative to the flow scale to be varied while maintaining constant data acquisition parameters and a similar pressure at the impingement location. Table 1 summarizes the specifications of the jets.

Table 1: Parameter space of the experiment

\begin{tabular}{ccccc}
\hline$U_{\max }(\mathrm{m} / \mathrm{s})$ & Frequency, $f(1 / \mathrm{s})$ & $\begin{array}{c}\text { Displacement } \\
\text { amplitude } \\
L_{0}(\mathrm{~mm})\end{array}$ & $\begin{array}{c}\text { Dynamic Pressure } \\
\text { Change }(\mathrm{Pa})\end{array}$ & $\begin{array}{c}\text { Orifice Diameter, } \\
D_{0}(\mathrm{~mm})\end{array}$ \\
\hline 2 & 2.5 & 80 & 750 & 4 \\
2 & 5 & 40 & 750 & 4 \\
2 & 10 & 20 & 750 & 4 \\
\hline
\end{tabular}

\section{Results and Discussion}

\subsection{Effect of the Boundary Condition Implementation}

To evaluate boundary condition options for each jet, the pressure field was computed by providing the pressure from the $\mathrm{S} 2$ sensor at the initial time step only and also by providing the time-averaged pressure from the S2 sensor at every time step, which is reasonably steady (table 2). The former results in pure Neumann type boundary condition for every time step subsequent to the initial time step and the latter results in mixed boundary conditions for all the time steps. For both conditions, the pressure is calculated 
with convection velocity estimation turned on and off. This results in four experimental cases which are summarized in table 3 .

Table 2: Mean and standard deviation of S2 sensor pressure data for different jets

\begin{tabular}{ccc}
$\begin{array}{c}\text { Jet displacement } \\
\text { amplitude }(\mathrm{mm})\end{array}$ & Mean Pressure (Pa) & Standard deviation of pressure (Pa) \\
\hline 80 & 0 & 93 \\
40 & 0 & 95 \\
20 & 0 & 84 \\
\hline
\end{tabular}

Table 3: Experimental cases

\begin{tabular}{ccccc}
\hline Case & S2 Boundary Condition & Convection Estimation & Boundary Condition type & Line color \\
\hline & & & & mixed \\
1 & Constant & off & mixed & red \\
2 & Constant & on & Neumann & orange \\
3 & Initial & off & Neumann & black \\
4 & Initial & on &
\end{tabular}

The pressure data from the S1 sensor and pressure calculated from PIV at the impingement location for each jet using these different boundary conditions are plotted in figure 3 . It can be observed that when the convection velocity estimation is turned on, both case 2 and case 4 show a temporal drift. The pressure calculated without the convection velocity estimate (case 1 and case 3 ) does not show any temporal drift, however the computed pressure at the impingement location exhibits more random error. Also the constant S2 boundary condition case (mixed type boundary condition) with convection velocity estimate turned on shows a drift similar to the initial S2 boundary condition (pure Neumann condition) case with convection velocity estimate turned on. Furthermore, the temporal drift increase with decreasing displacement amplitude of the jet. 


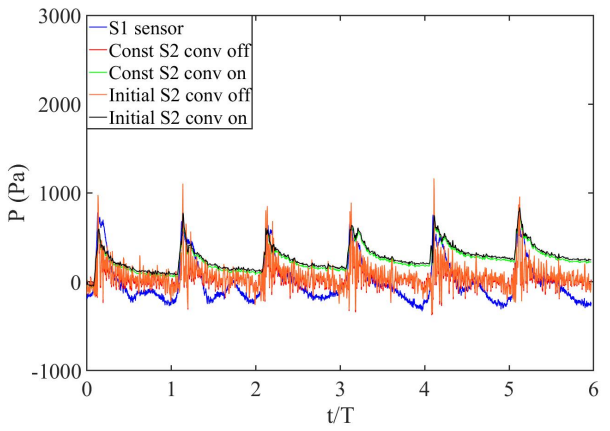

(a)

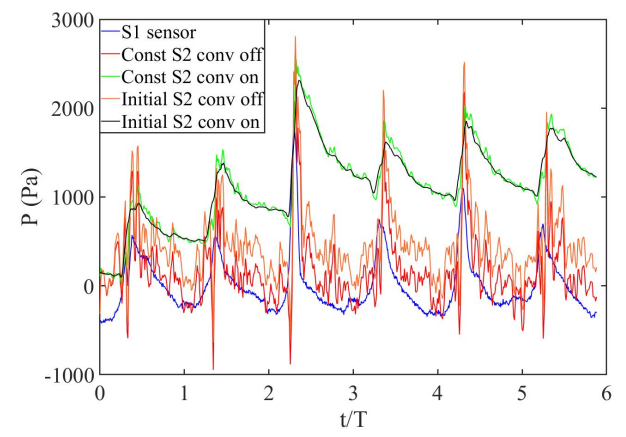

(c)

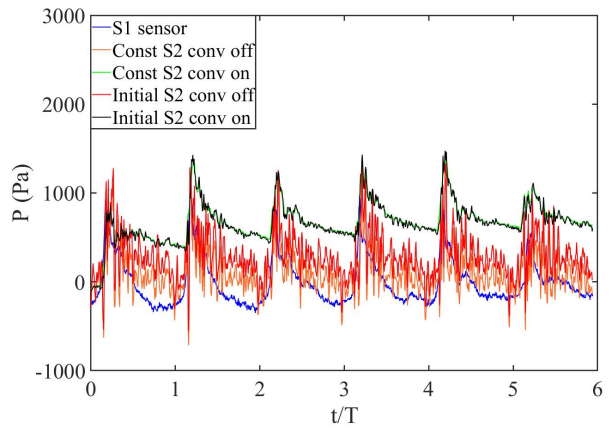

(b)

Figure 3: Pressure from PIV for a synthetic jet for three different displacement amplitudes and frequencies but similar dynamic pressure change. a) $L_{0}=80 \mathrm{~mm}, f=2.5 \mathrm{~Hz}$, b) $L_{0}=40 \mathrm{~mm}, f=5 \mathrm{~Hz}$, c) $L_{0}=20 \mathrm{~mm}$, $f=10 \mathrm{~Hz}$. The impingement pressure is $750 \mathrm{~Pa}$ for all three cases. The numeric value of $\xi$ for convection velocity estimate on cases and convection velocity estimate off cases are 1 and 0.0001 respectively.

\subsection{Pressure from PIV and Temporal Resolution}

Figure 3 also shows that when the convection velocity is used, the temporal drift increases with increasing displacement amplitude of the synthetic jet. Since these data were obtained at the same acquisition rate, this trend indicates that the calculated pressure field is related to the effective temporal resolution of the PIV data. To systematically investigate this trend, the pressure field was recalculated in a way so that all three jets have the same number of velocity fields per cycle. This reduces the temporal resolution of the $L_{0}=80$ and $L_{0}=40$ jets by a fourth and a half, respectively, yielding an equivalent relative temporal resolution for all three jets. Figure 4 shows the pressure at the impingement location from the S1 sensor and from PIV for all three jets. The reduced temporal resolution in the $L_{0}=80$ and $L_{0}=40$ jets results in a higher temporal drift. However, even with the same effective temporal resolution, the temporal drift still increases with decreasing displacement amplitude.

\subsection{Effect of Spatial Resolution}

In order to investigate the effect of spatial resolution on the calculated pressure field, the PIV images were reprocessed with a larger final pass interrogation window size of $40 \times 40 \times 40$ voxels which results in a coarser spatial resolution. Figure 5 shows the pressure from PIV for reduced spatial resolution for all three jets. It can be observed that the reduced spatial resolution lowers the temporal drift. 


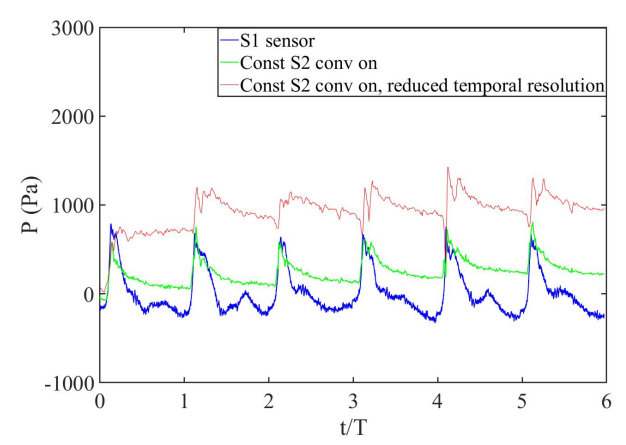

(a)

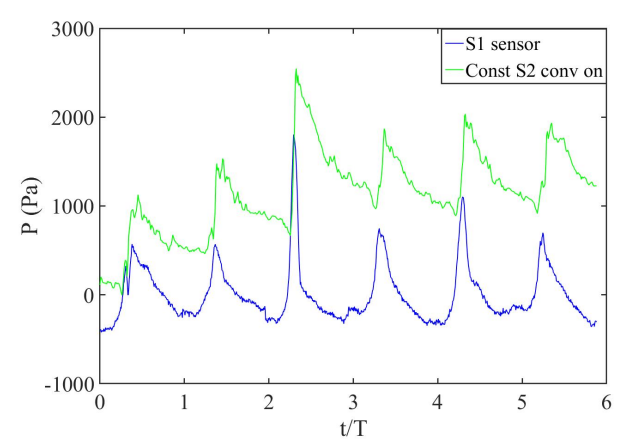

(c)

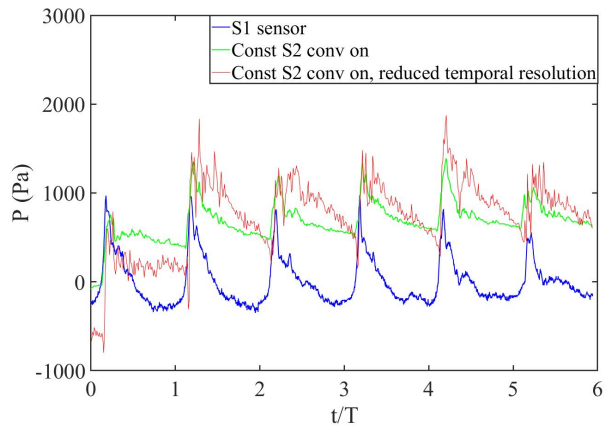

(b)

Figure 4: Effect of temporal resolution on the pressure calculated from PIV with 98 vector fields for each case. a) $L_{0}=80 \mathrm{~mm}, f=2.5 \mathrm{~Hz}$, b) $L_{0}=40 \mathrm{~mm}, f=5$, c) $L_{0}=20 \mathrm{~mm}, f=10 \mathrm{~Hz}$.

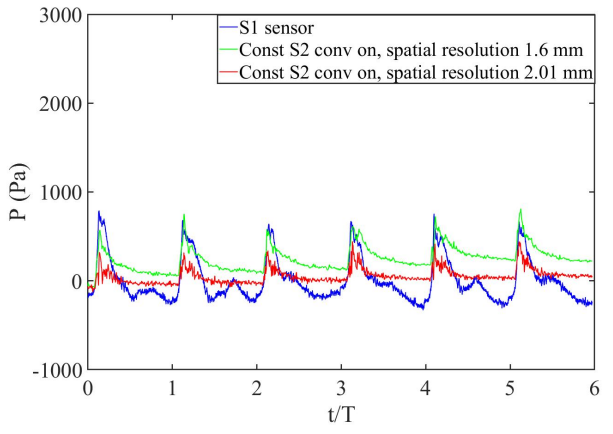

(a)

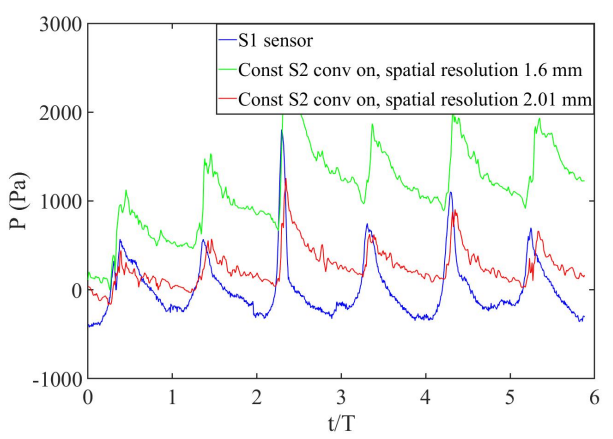

(c)

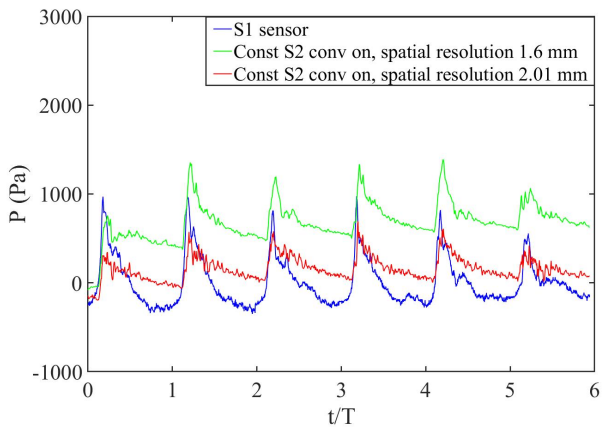

(b)

Figure 5: Effect of reduced spatial resolution on the pressure from PIV. a) $\left.L_{0}=80 \mathrm{~mm}, f=2.5 \mathrm{~Hz}, \mathrm{~b}\right) L_{0}=$ $40 \mathrm{~mm}, f=5, \mathrm{c}) L_{0}=20 \mathrm{~mm}, f=10 \mathrm{~Hz}$ 


\section{Conclusion and Future Work}

In this study, the pressure field for an impinging synthetic jet is reconstructed from PIV velocity fields using a Poisson equation-based pressure solver. The solver has the option to estimate the temporal derivative of the pressure for time resolved PIV data via estimation of the convection velocity of flow structures. The pressure solver can also reconstruct the pressure field from individual velocity fields without considering the time derivative of the pressure which is similar to traditional 3D Poisson solvers. The reconstructed pressure with the estimation of the time derivative of the pressure demonstrates a temporal drift. While the pressure without the temporal derivative estimation does not show any drift, it exhibits much larger random errors.

The reconstructed pressure also shows sensitivity to the temporal resolution of the PIV data and the spatial resolution of the PIV processing. This current study shows that the temporal drift in the reconstructed pressure is lower for a coarse spatial resolution. Future work will include the extension of the parameter space to identify any optimal spatial resolution for the pressure reconstruction as described in Pan et al. (2018). Pressure field reconstruction from the velocity field obtained by cutting edge particle tracking methods, such as Shake the Box, (STB) (Schanz et al., 2016) and the study of the relevant parameters on the pressure field will be included in future studies. Furthermore, as the uncertainty quantification of the STB velocity field becomes available, the propagation of velocity field uncertainty into the pressure field and quantify the uncertainty in the reconstructed pressure field will become possible.

\section{Acknowledgements}

Notice: This report was prepared as an account of work sponsored by an agency of the United States Government. Neither the United States Government nor any agency thereof, nor any of their employees, nor any of their contractors, subcontractors or their employees, makes any warranty, express or implied, or assumes any legal liability or responsibility for the accuracy, completeness, or any third partys use or the results of such use of any information, apparatus, product, or process disclosed, or represents that its use would not infringe privately owned rights. Reference herein to any specific commercial product, process, or service by trade name, trademark, manufacturer, or otherwise, does not necessarily constitute or imply its endorsement, recommendation, or favoring by the United States Government or any agency thereof or its contractors or subcontractors. The views and opinions of authors expressed herein do not necessarily state or reflect those of the United States Government or any agency thereof. The authors would like to thank Dr. Young Jin Jeon of LaVision $\mathrm{GmbH}$ for his assistance in understanding the equations that are solved within DaVis.

\section{References}

Bark DL, Johnson B, Garrity D, and Dasi LP (2017) Valveless pumping mechanics of the embryonic heart during cardiac looping: Pressure and flow through micro-PIV. Journal of Biomechanics 50:50-55

Baur T and Kongeter J (1999) PIV with high temporal resolution for the determination of local pressure reductions from coherent turbulence phenomena. in 3rd International Workshop on Particle Image Velocimetry. Santa Barbara, CA, USA

De Kat R and Ganapathisubramani B (2013) Pressure from particle image velocimetry for convective flows: A Taylor's hypothesis approach. Measurement Science and Technology 24

Elsinga GE, Scarano F, Wieneke B, and Van Oudheusden BW (2006) Tomographic particle image velocimetry. Experiments in Fluids 41:933-947

Ghaemi S, Ragni D, and Scarano F (2012) PIV-based pressure fluctuations in the turbulent boundary layer. Experiments in Fluids 53:1823-1840

Jensen A, Sveen JK, Grue J, Richon JB, and Gray C (2001) Accelerations in water waves by extended particle image velocimetry. Experiments in Fluids 30:500-510

Jeon YJ, Michaelis D, and Wieneke B (2017) Estimation of flow structure transport in TR-PIV data and its application to pressure field evaluation. in 2nd Workshop on Data Assimilation and CFD Processing for PIV and Lagrangian Particle Tracking. Delft, The Netherlands 
Laskari A, de Kat R, and Ganapathisubramani B (2016) Full-field pressure from snapshot and time-resolved volumetric PIV. Experiments in Fluids 57:1-14

Lignarolo LE, Ragni D, Krishnaswami C, Chen Q, Simão Ferreira CJ, and van Bussel GJ (2014) Experimental analysis of the wake of a horizontal-axis wind-turbine model. Renewable Energy 70:31-46

Liu X and Katz J (2004) Measurements of pressure distribution in a cavity flow by integrating the material acceleration. Proceedings of the ASME Heat Transfer/Fluids Engineering Summer Conference 2004, HT/FED 2004 3:621-631

Liu X and Katz J (2006) Instantaneous pressure and material acceleration measurements using a fourexposure PIV system. Experiments in Fluids 41:227-240

Oren L, Gutmark E, and Khosla S (2015) Intraglottal velocity and pressure measurements in a hemilarynx model. The Journal of the Acoustical Society of America 137:935-943

Pan Z, Whitehead J, Thomson S, and Truscott T (2016) Error propagation dynamics of PIV-based pressure field calculations: How well does the pressure Poisson solver perform inherently?. Measurement Science and Technology 27

Pan Z, Whitehead JP, Richards G, Truscott TT, and Smith BL (2018) Error propagation dynamics of PIVbased pressure field calculation (3): What is the minimum resolvable pressure in a reconstructed field?. arXiv pages $1-23$

Schanz D, Gesemann S, and Schröder A (2016) Shake-The-Box: Lagrangian particle tracking at high particle image densities. Experiments in Fluids 57:1-27

Schneiders JF, Pröbsting S, Dwight RP, van Oudheusden BW, and Scarano F (2016) Pressure estimation from single-snapshot tomographic PIV in a turbulent boundary layer. Experiments in Fluids 57:1-14

Tsuji Y, Fransson JHM, Alfredsson PH, and Johansson AV (2007) Pressure statistics and their scaling in high-Reynolds-number turbulent boundary layers. Journal of Fluid Mechanics 585:1-40

Van Oudheusden BW (2013) PIV-based pressure measurement. Measurement Science and Technology 24

Villegas A and Diez FJ (2014) On the quasi-instantaneous aerodynamic load and pressure field measurements on turbines by non-intrusive PIV. Renewable Energy 63:181-193

Violato D, Moore P, and Scarano F (2011) Lagrangian and Eulerian pressure field evaluation of rod-airfoil flow from time-resolved tomographic PIV. Experiments in Fluids 50:1057-1070 